\title{
Biosynthesis and characterization of silver nanoparticles using ginger spent and their antibacterial activity
}

\author{
Sinthia Ganesan', Palanichamy Mehalingam²*, \\ Govindan Sadasivam Selvam ${ }^{3}$ \\ 'Department of Biotechnology, V.V. Vanniaperumal College for Women (Autonomous), Virudhunagar-626001, \\ Tamil Nadu, India, ${ }^{2}$ Research Department of Botany, V.H.N. Senthikumara Nadar College (Autonomous), \\ Virudhunagar-626001, Tamil Nadu, India, 3'Department of Biochemistry, School of Biological Sciences, Madurai \\ Kamaraj University, Madurai - 625 021, Tamil Nadu, India
}

*Corresponding Author: Palanichamy Mehalingam, E-mail: mehalingamp@gmail. com

Received: November 22, 2020 Revised: August 23, 2021

Accepted: August 24, 2021

Published: September 20, 2021

\begin{abstract}
Ginger spent is the byproduct of spice industries that remove the essential oils of ginger (Zingiber officinale) for the food industry and medicinal purposes. Ginger is a well known spice used often for seasoning in Indian cuisine. The deoiled ginger has no specific use mostly goes to waste. Hence, we utilized this industrial waste product in the efficient synthesis of silver nanoparticles with the aid of UV irradiation from a solution of $1 \mathrm{mM}$ silver nitrate and spent extract in the ratio 9:1. Immediate colour change from pale yellow to dark brown was noted indicating the rapid synthesis of silver nanoparticles. These nanoparticles were centrifuged, dried and well characterized. UV Vis Spectroscopy, XRD analysis, Zeta potential and SEM analysis were carried out. It was commendable that the size of the nanoparticles fell well within the upper limit of $100 \mathrm{~nm}$. Agar well diffusion method was used to screen the antimicrobial activity of the well characterized silver nanoparticles. They were tested against seven pathogenic strains of three Gram negative bacteria (Escherichia coli, Klebsiella pneumonia and Pseudomonas aeruginosa) three Gram positive bacteria (Bacillus subtilis, Staphylococcus aureus and Streptococcus faecalis) and a fungus (Candida albicans). It was seen that the zone of inhibition (ZOI) in the well plate method increased on increasing the concentration of silver nanoparticles. Further studies could lead to the application of these silver nanoparticles in the field of nanomedicine.
\end{abstract}

KEYWORDS: Antimicrobial activity; Ginger spent; UV irradiation; Zingiber officinale

\section{INTRODUCTION}

The field of nanoscience shows the good scope and is growing rapidly with several applications. Current research is aimed at developing new ways to efficiently scale up the synthesis of nanoparticles (NPs). There is huge demand for metallic nanoparticles produced from noble metals like gold, platinum and silver since they are inert and hence find several applications in therapeutics and biomedical devices. These silver nanoparticles are in demand as they exhibit surface Plasmon resonance (SPR) in the visible light region (Bhakya et al., 2016).Zingiber officinale L. commonly known as ginger is a member of the Zingiberaceae family and is a flowering monocot. The edible portion is mainly the rhizome or root of the plant. Ginger is a good antibacterial agent and finds application in the ayurvedic systems of medicine since time immemorial. Most Asian countries like India use it to add spice to their daily cuisine and also as a handy remedy for treating cold, cough and indigestion problems (Sharma \& Bhagatwala, 2019).
The essential oils present in ginger, namely, gingerol and zingiberine are extracted by distillation from the underground rhizome of ginger. Ginger oil has a unique smell that is spicy, strong and hot. The concentrated oil has a huge number of applications that include aromatherapy, use as a skin and hair care product and of course the universal use in cuisine worldwide to yield a distinct pungency to food and food products. The essential oil of ginger is immensely beneficial to human health as it is a proven antioxidant that scavenges free radicals. Its use is also reported in the treatment of arthritis and other acute or chronic inflammatory conditions to give pain relief. Hence we know that ginger oil is well established as an anti-oxidant, anti-inflammatory as well as anti-nociceptive agent (Jeena et al., 2013). Various methods are available to synthesize nanoparticles which can be broadly classified into Chemical, Physical and Biological means. Of these, biological process is the most viable option as chemical methods involve the use of highly expensive as well as toxic substances that pollute the environment, while

Copyright: (C) The authors. This article is open access and licensed under the terms of the Creative Commons Attribution License (http://creativecommons.org/licenses/by/4.0/) which permits unrestricted, use, distribution and reproduction in any medium, or format for any purpose, even commercially provided the work is properly cited. Attribution — You must give appropriate credit, provide a link to the license, and indicate if changes were made. 
physical processes require sophisticated and costly equipment. Hence, bio-inspired green synthesis is growing rapidly into an important branch in nanoscience. Nanoparticles may be formed from scratch by precipitation in a colloidal solution (bottom-up) or by breaking down large substances to nanosize (top-down). The bottom-up approach is applied to chemical and biological methods, while physical methods rely largely on the top-down approach to break down larger particles by various techniques like grinding, laser ablation or milling (Ahmed et al., 2016). Several studies are available that indicate the pharmacological importance and physiological activities of ginger and its essential oils. Ginger oil is of importance in the pharmaceutical industry and in high demand. Hence, several spice processing industries extract the oil of ginger to supply food and medical fields. This leaves the de-oiled ginger (spent) as an unnecessary byproduct often used as an adulterant in low grade food products. The present study is aimed at the efficient use of aqueous extracts of this industrial waste (ginger spent) to aid the green synthesis of silver nanoparticles. These biosynthesized silver nanoparticles have been well characterized for antimicrobial activity indicating their application in nanomedicine.

\section{MATERIALS AND METHODS}

\section{Materials}

Raw material (Ginger spent or De-oiled Zingiber officinale) (Figure 1.) was procured from the world's largest manufacturer of natural spice powders and value added spice extracts, Synthite Industries Private Ltd., Kochi. Chemical reagents of high quality analytical grade were purchased and directly used without further purification. The silver nitrate used for the experiments was bought from Sigma-Aldrich, India. The culture medium used for the study, namely Muller Hilton Agar and Broth were brought from Hi-Media, Mumbai. All the seven strains of microorganisms used for screening of antimicrobial activity were donated by Gandhigram Rural Institute, Gandhigram. All steps mentioned below were carried out only with double distilled water.

\section{Preparation of Extracts}

The spent ginger sample was sun dried to remove moisture, repacked and stored in a refrigerator until further use. To prepare the aqueous extract, $10 \mathrm{gm}$ of powder was weighed into a $500 \mathrm{ml}$ beaker and $100 \mathrm{ml}$ of double distilled water was added to it. The mixture was stirred thoroughly and heated to a temperature of $60{ }^{\circ} \mathrm{C}$ for 15 minutes. The extract was then obtained by filtering the resulting solution through Whatman No.l filter paper. The aqueous extract (Figure 2.) so obtained was used immediately in the synthesis of silver nanoparticles (Pattabiraman et al., 2016).

\section{Silver Nanoparticles Biosynthesis}

$100 \mathrm{ml}$ of $1 \mathrm{mM}$ silver nitrate solution was first prepared in a standard flask. To $90 \mathrm{ml}$ of silver nitrate solution taken in an Erlenmeyer flask, $10 \mathrm{ml}$ of the prepared aqueous extract was

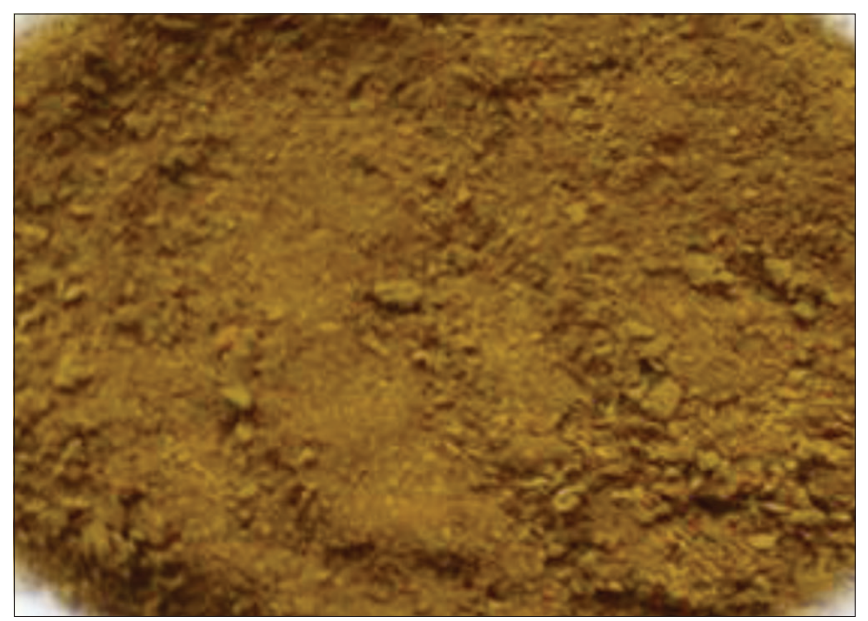

Figure 1: De-oiled Zingiber officinale

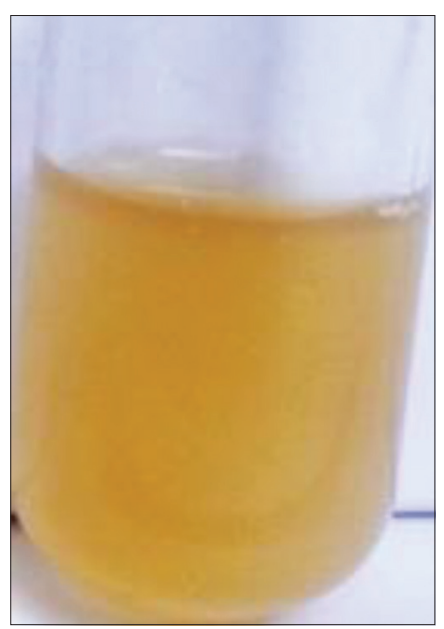

Figure 2: De-oiled Zingiber officinale extract

added and mixed evenly. The mixture was closed and placed in sunlight. After 30 minutes it was observed that the solution changed colour from pale yellow to dark brown. This colour change indicates the formation of silver nanoparticles.

\section{Characterization of Biosynthesized Silver Nanoparticles}

The formation of silver nanoparticles was confirmed by studying the optical properties of the solution using a UV visible spectroscope (UV 1800V of Shimadzu, Japan). A Flow cytometer was used to determine the stability of the silver nanoparticles by measuring the Zeta potential of the solution. A particle size analyzer was used to study the size and surface morphology of the newly synthesized silver nanoparticles. The functional groups involved in stabilizing the silver nanoparticles were identified using FTIR spectroscopy (Thermo Scientific Nicolet 380) by KBr pellet method. EDAX analysis was used to check the purity of silver present in the silver nanoparticles. XRD analysis (by using a Diffractometer by Shimadzu, Japan) was carried out at $30 \mathrm{~mA}, 40 \mathrm{kV}$ using $\mathrm{Cu}$ $\mathrm{ka}$ radiations for a $2 \theta$ angle to study the crystalline structure of the silver nanoparticles. 


\section{Antimicrobial Activity}

Agar well diffusion method (Valgas et al., 2007) was followed to screen the biosynthesized silver nanoparticles for antimicrobial efficiency against seven human pathogens (Bacillus subtilis, Candida albicans, Escherichia coli, Klebsiella pneumoniae, Pseudomonas aeruginosa, Staphylococcus aureus and Streptococcus faecalis). The microorganisms were subcultured in Muller Hilton Agar (MHA) and incubated overnight at $37^{\circ} \mathrm{C}$. The culture incubated for 24 hours was spread plated on MHA in Petri plates. Six wells were bored on the gel with a cork borer of diameter $6 \mathrm{~mm}$ in a circle with one well in the centre to load antibiotic, Streptomycin $(20 \mu \mathrm{g} /$ $\mathrm{ml}$ ) which functions as control. The other five wells were loaded with the prepared extract, pure silver nitrate and three concentrations of prepared silver nanoparticles $(100 \mu \mathrm{g} / \mathrm{ml}$, $150 \mu \mathrm{g} / \mathrm{ml}$ and $200 \mu \mathrm{g} / \mathrm{ml}$ ) clockwise from top. The plates were again incubated overnight at room temperature. The zone of inhibition (ZOI) formed around the wells were measured in $\mathrm{mm}$ and results were tabulated to compare the antimicrobial activity of the silver nanoparticles with that of pure extract, silver nitrate and antibiotic.

\section{RESULTS}

Initially, a pale yellow solution resulted when an aqueous extract of ginger spent was added to a colourless solution of silver nitrate. When exposed to sunlight, the colour of the solution gradually changed to dark brown as seen in the picture below (Figure 3). It was noted that this colour change was a slow process when kept overnight in a dark room but immediately visible when exposed to sunlight. This confirms the photo catalytic effect of UV irradiation and its supportive role in the formation of silver nanoparticles.

\section{Characterization of Silver Nanoparticles}

\section{Optical properties}

Metallic silver shows a characteristic peak at $420 \mathrm{~nm}$ due to Surface Plasmon Resonance (SPR) caused by vibration of surface electrons. This property is used to confirm the formation of silver nanoparticles using a UV visible spectroscope. The UV spectra revealed three peaks as shown in the graph below (Figure 4) of which peak 1 at $420 \mathrm{~nm}$ corresponds to that of silver nanoparticles.

\section{Fourier Transform Infrared Spectroscopy}

The chemical groups responsible for the reduction and stabilization of the green synthesized silver nanoparticles were identified by FTIR analysis. The spectrum showed several peaks as shown in the graph below (Figure 5). The five major peaks are at $1028.95 \mathrm{~cm}^{-1}, 1379.97 \mathrm{~cm}^{-1}, 1601.77 \mathrm{~cm}^{-1}$, $2360.71 \mathrm{~cm}^{-1}$ and $3421.48 \mathrm{~cm}^{-1}$. There are also five minor peaks at $533.28 \mathrm{~cm}^{-1}, 773.4 \mathrm{~cm}^{-1}, 1227.61 \mathrm{~cm}^{-1}, 1310.54 \mathrm{~cm}^{-1}$ and $2924.85 \mathrm{~cm}^{-1}$. The largest narrow peak at $2360.71 \mathrm{~cm}^{-1}$ is of the same absorbance as that of $\mathrm{CO}_{2}$. The next biggest

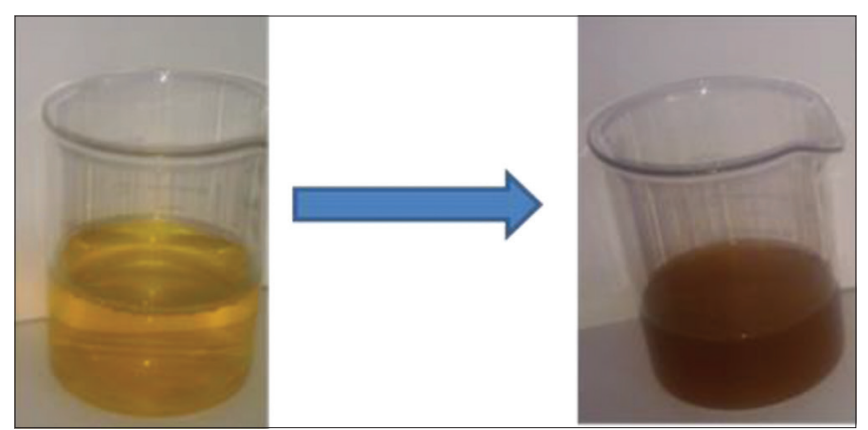

Figure 3: Formation of Silver Nanoparticles using Ginger spent extract

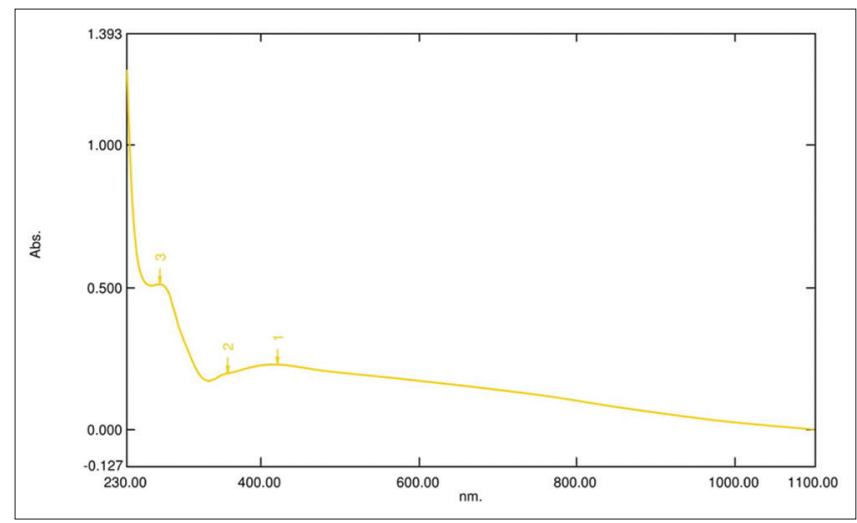

Figure 4: UV spectra- peak 1 at $420 \mathrm{~nm}$ indicates presence of silver metal

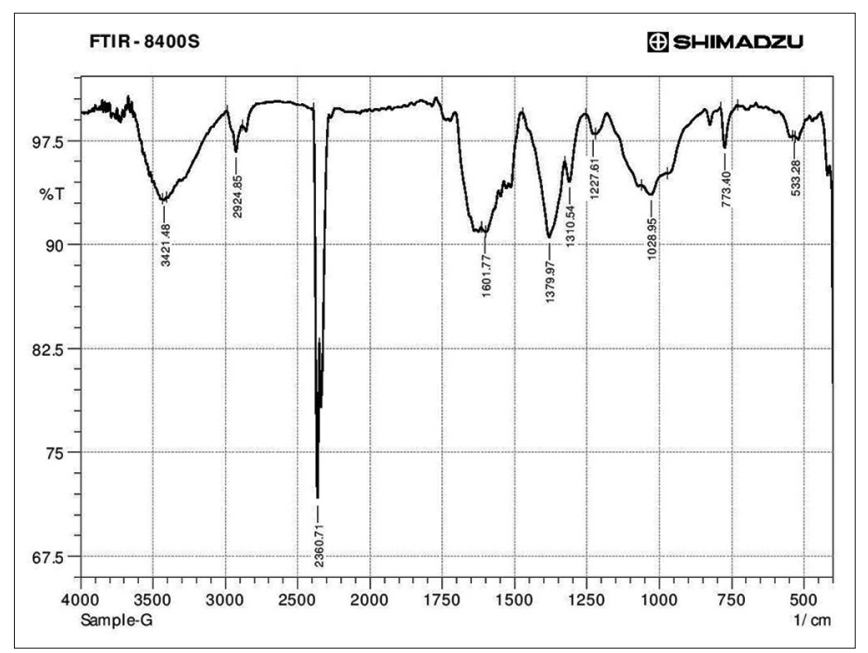

Figure 5: FTIR spectra of silver nanoparticles indicates presence of stabilizing groups

peak is at 1379.97 which corresponds to that of aliphatic nitro compounds. The next two peaks at $3421.48 \mathrm{~cm}^{-1}$ and $1601.7 \mathrm{~cm}^{-1}$ can be assigned to that of primary amines while the overlapping peaks at $1028.95 \mathrm{~cm}^{-1}$ indicate the presence of aliphatic amines and the one at $1310.54 \mathrm{~cm}^{-1}$ belongs to aromatic amines. The smaller peak at $533.28 \mathrm{~cm}^{-1}$ shows bromoalkanes, $773.40 \mathrm{~cm}^{-1}$ reveals aromatic benzene ring, $1227.61 \mathrm{~cm}^{-1}$ is for aromatic ethers and $2924.85 \mathrm{~cm}^{-1}$ shows the presence of ammonium ions (N-H bond) (Sanati \& Andersson, 1993). 


\section{X-Ray Diffraction Studies}

XRD data indicates the crystalline shape and size of the silver nanoparticles. The size can be calculated using the following equation by Holzwarth and Gibson (2011):

$$
\tau=K \lambda / \beta \cos \theta
$$

where:

- $\tau$ is the calculated crystal size measured in nanometers.

- $K$ is the shape factor - a dimenionless value close to unity. It is often taken as 0.9 but may vary slightly depending on the crystal shape.

- $\lambda$ is the wavelength of X-ray.

- $\beta$ often referred to as $2 \theta$ is the full width half maximum(FWHM)

- and $\theta$ is Bragg's angle

With the use of XRD tabulated data in Table 1 and this equation, the average crystal size was calculated as $22.7 \mathrm{~nm}$.

The major peaks are seen at 32.35, 38.27 and 46.26 as shown in the graph (Figure 6) below. The d-spacing values were compared to standard values in JCPDF software to arrive at the crystal structure. It was seen that the values matched PDF\#411402 which corresponds to hexagonal crystal structure corresponding to $112(1.386)$ and 201(1.2400) planes.

\section{Particle Size Analyzer (SPS)}

A Flow cell cytometer (Shimadzu SALD-2300) was used to determine the exact grain size of the newly synthesized nanoparticles. It was seen that their size varied from $85 \mathrm{~nm}$ to $120 \mathrm{~nm}$ with most particles averaging a grain size of $96 \mathrm{~nm}$ (Figure 7). The increase in grain size, compared to crystal size measured using XRD data, could be attributed to functional groups around the particles forming a free floating layer in solution.

\section{Zeta Potential}

The same instrument was used in calculating the zeta potential of the nanoparticles. The silver nanoparticles showed good stability in solution with a negative value of $-31 \mathrm{mV}$ as shown in Figure 8 (Clogston \& Patri, 2011).

\section{SEM Analysis}

The nanoparticles were studied using scanning electron microscope to confirm the size and shape of the nanoparticles. It can be seen that the particles varied in size with most falling within $100 \mathrm{~nm}$ as indicated with red lines in the figure below (Figure 9). The particles showed crystalline shapes.

\section{Antimicrobial Activity of Silver Nanoparticles}

Silver nanoparticles are highly effective in controlling the growth of bacteria and fungi and these hybrid nanoparticles

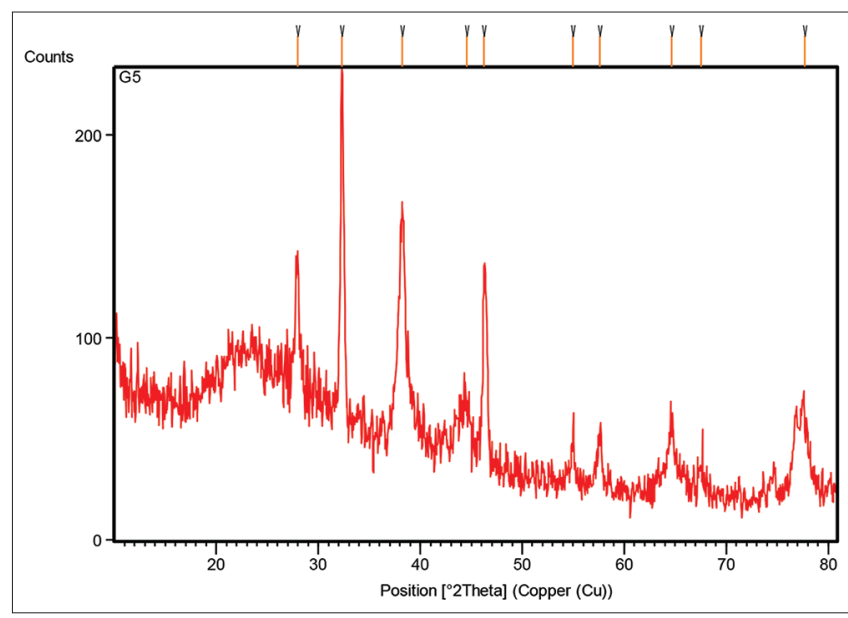

Figure 6: XRD of silver nanoparticles revealing hexagonal structure

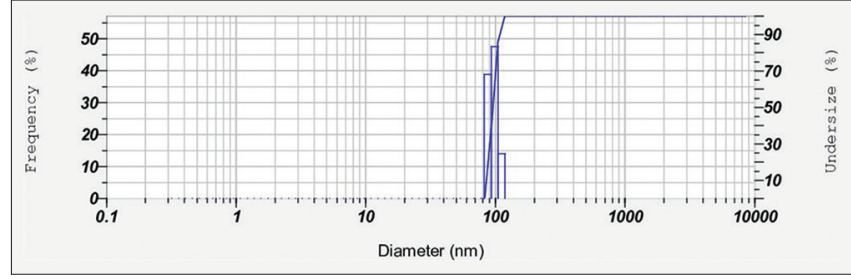

Figure 7: Particle Size Analysis of silver nanoparticles synthesized using Ginger spent

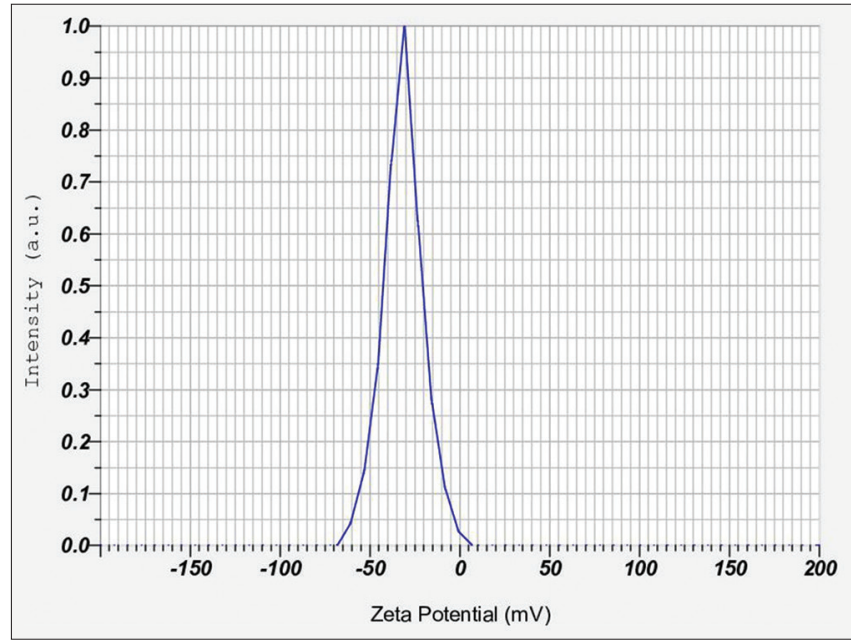

Figure 8: Zeta potential of silver nanoparticles synthesized using Ginger spent

Table 1: X-Ray Diffraction Studies

\begin{tabular}{|c|c|c|c|c|}
\hline Position [ $\left.{ }^{\circ} 2 \theta\right]$ & $\begin{array}{l}\text { Height } \\
\text { [cts] }\end{array}$ & FWHM Left $\left[{ }^{\circ} 2 \theta\right]$ & d-spacing $[\AA]$ & Rel. Int. [\%] \\
\hline 27.9948 & 67.93 & 0.2952 & 3.18730 & 39.28 \\
\hline 32.3459 & 172.94 & 0.2460 & 2.76780 & 100.00 \\
\hline 38.2731 & 112.25 & 0.3936 & 2.35171 & 64.91 \\
\hline 44.5249 & 28.47 & 0.5904 & 2.03494 & 16.46 \\
\hline 46.2613 & 95.95 & 0.2952 & 1.96253 & 55.48 \\
\hline 54.9780 & 25.77 & 0.2952 & 1.67022 & 14.90 \\
\hline 57.6254 & 27.04 & 0.3936 & 1.59963 & 15.63 \\
\hline 64.6199 & 36.90 & 0.4920 & 1.44235 & 21.34 \\
\hline 67.5399 & 12.01 & 0.4920 & 1.38693 & 6.94 \\
\hline 77.6495 & 41.88 & 1.1808 & 1.22968 & 24.21 \\
\hline
\end{tabular}




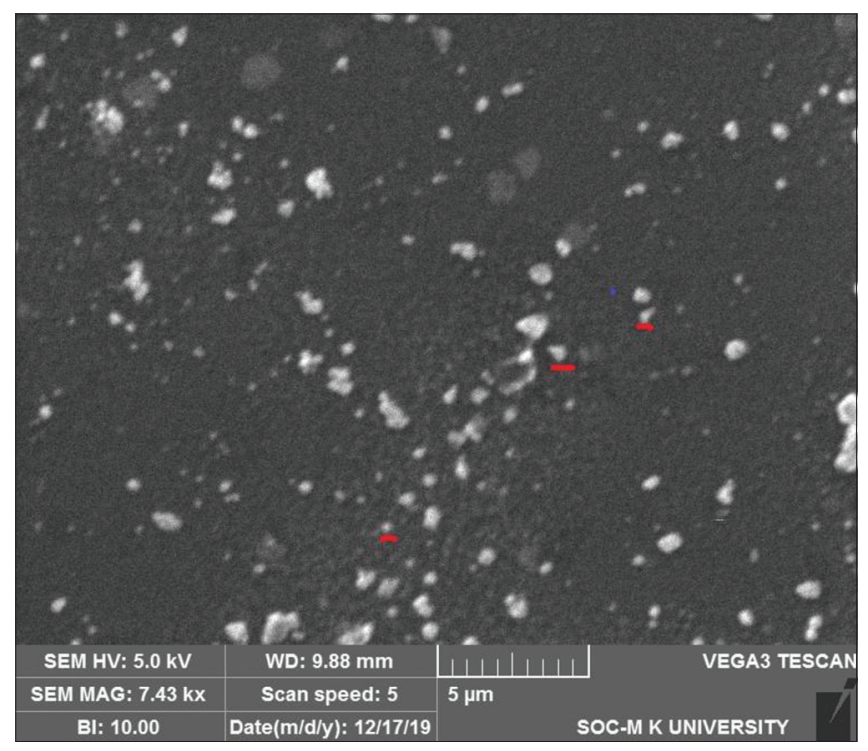

Figure 9: SEM image of silver nanoparticles synthesized using Ginger spent

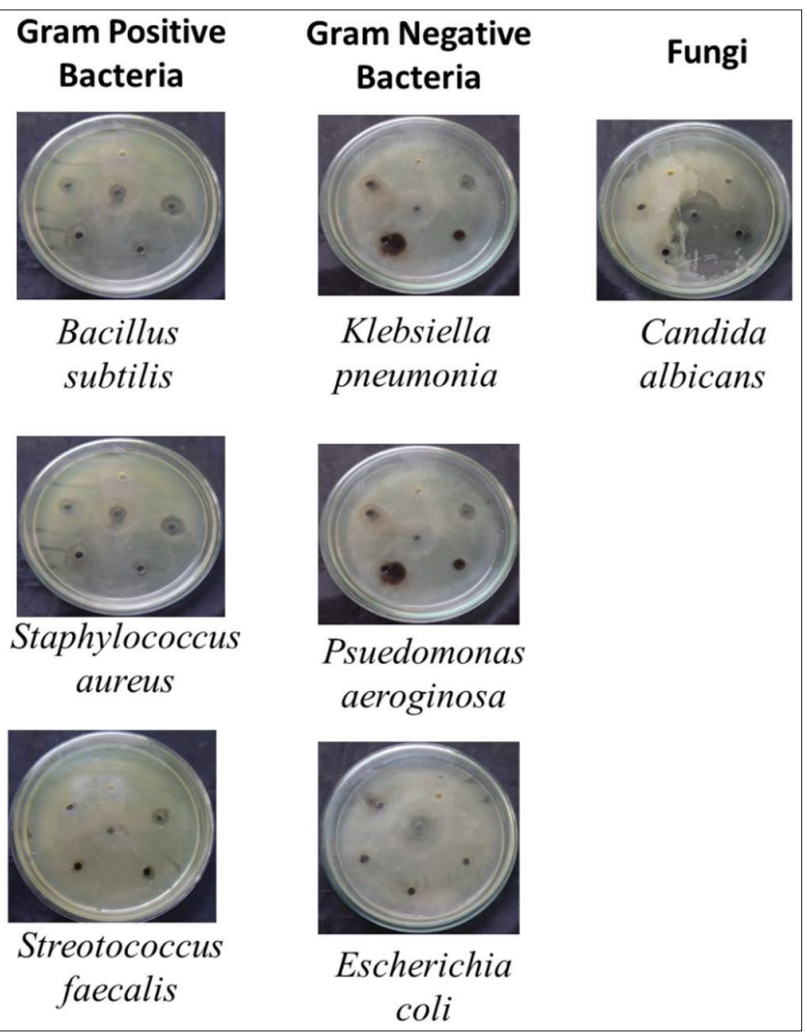

Figure 10: Antimicrobial activity by agar well plate method proved to be no different. The particles were screened for antimicrobial activity against seven human pathogens of which one is a fungus (Candida albicans), three are Gram negative bacteria (Escherichia coli, Klebsiella pneumonia and Pseudomonas aeruginosa) and three Gram positive bacteria (Staphylococcus aureus, Streptococcus faecalis and Bacillus subtilis) by agar well diffusion method as shown below (Figure 10 \& Table 2). The ZOI was calculated for each microbe with respect to plant extract, silver nitrate, and three different concentrations of silver nanoparticles and compared to that of standard antibiotics to arrive at an accurate value. It was seen that the AgNPs showed average activity against the pathogens with a maximum zone of inhibition as $13.0 \pm 1.0 \mathrm{~mm}$ seen against Bacillus subtilis and Pseudomonas aeruginosa equally. The activity naturally increased with increasing concentrations of silver nanoparticles. All the microbes were fairly susceptible to the AgNPs paving the way for its significant use in the reduction of microbial populations where necessary.

\section{DISCUSSION}

Dawadi et al. (2021) and Garibo et al. (2020) noted that polyphenols, alkaloids and other antioxidants present in plant extracts function as reducing as well as capping agents in the synthesis of silver nanoparticles from silver nitrate. Also, reaction temperature, $\mathrm{PH}$ and ratio of salt: plant extract all control the size of the green synthesized silver nanoparticles. Silver nanoparticles find applications as water purifiers, pesticides and novel drugs due to their efficient antimicrobial action. The toxicity of the silver nanoparticles needs to be studied before further use in large scale. Previously, Roy et al. (2019) showed that green synthesized silver nanoparticles show enhanced antimicrobial activity due to the combined effect of silver and plant antioxidants and can hence be used to treat multidrug resistant bacteria by easily disrupting their membranes due to this synergistic effect. In 2020, Mangindaana and coworkers were able to synthesize silver nanoparticles using spent coffee grounds, as reducing and capping agents, which are considered a waste product of the coffee industry but are actually rich in biofunctional molecules.

In the present study, FTIR analysis of the silver nanoparticles revealed the presence of phenols which could function as reducing agents. The silver nanoparticles showed better antimicrobial activity than silver nitrate alone or spent extract alone (Table 2) proving the synergistic effect. Ginger spent contains traces of residual polyphenols which are sufficient to

Table 2: Mean Zone Of Inhibition (in $\mathrm{mm}$ ) of Silver nanoparticles synthesized using Ginger spent extract

\begin{tabular}{|c|c|c|c|c|c|c|c|}
\hline S. No & Cultures & Ginger spent extract $(10 \mu \mathrm{l})$ & AgN03 $(10 \mu \mathrm{l})$ & $\operatorname{AgNPs}(10 \mu \mathrm{l})$ & $\operatorname{AgNPs}(20 \mu \mathrm{l})$ & $\operatorname{AgNPs}(30 \mu \mathrm{l})$ & Streptomycin $(10 \mu \mathrm{l})$ \\
\hline 1. & Bacillus subtilis & $8 \pm 0.6$ & $9 \pm 0.7$ & $11 \pm 1.0$ & $13 \pm 0.6$ & $13 \pm 1.0$ & $27 \pm 1.2$ \\
\hline 2. & Staphylococcus aureus & $7 \pm 1.0$ & $8 \pm 0.3$ & $8 \pm 0.5$ & $10 \pm 0.5$ & $11 \pm 0.7$ & $25 \pm 0.6$ \\
\hline 3. & Streptococcus faecalis & $8 \pm 0.3$ & $8 \pm 0.5$ & $9 \pm 0.4$ & $9 \pm 0.6$ & $10 \pm 1.0$ & $20 \pm 1.0$ \\
\hline 4. & Klebsiella pneumoniae & $9 \pm 1.0$ & $8 \pm 1.2$ & $9 \pm 0.7$ & $11 \pm 0.8$ & $12 \pm 0.6$ & $21 \pm 1.0$ \\
\hline 5. & Pseudomonas aeruginosa & $8 \pm 0.7$ & $10 \pm 1.0$ & $12 \pm 0.4$ & $12 \pm 0.9$ & $13 \pm 1.3$ & $20 \pm 0.7$ \\
\hline 6. & E.coli & $7 \pm 0.5$ & $8 \pm 0.4$ & $8 \pm 0.5$ & $10 \pm 0.6$ & $10 \pm 0.7$ & $22 \pm 0.6$ \\
\hline 7. & Candida albicans & $6 \pm 0.4$ & $8 \pm 0.6$ & $9 \pm 0.3$ & $9 \pm 1.0$ & $11 \pm 0.5$ & $25 \pm 0.8$ \\
\hline
\end{tabular}


reduce silver nitrate to efficiently synthesize silver nanoparticles and can be used in large scale processes.

\section{CONCLUSION}

We can conclude that there are several other active ingredients present in ginger beyond Zingiberine and Gingerol that have antimicrobial activity since even the ginger spent was effectively able to control the growth of several microorganisms. Rapid synthesis of silver nanoparticles using ginger spent as stabilizing agent and sunlight as catalyst was feasible and the silver nanoparticles so synthesized were well characterized. This was done using UV Visible Spectroscopy, FTIR, XRD studies, SPS and SEM analysis. It was seen that the silver nanoparticles were hexagonal with an average size of $96 \mathrm{~nm}$. The particles were stabilized mostly by nitro groups. They showed moderate stability with a negative zeta potential of $-31.6 \mathrm{mV}$. The AgNPs were fairly active against the screened bacteria and fungi. An increase in the concentration of the silver nanoparticles resulted in an increase in antimicrobial activity. Best antibacterial activity was seen against Streptococcus faecalis and was equal to that of Streptomycin. This effective use of hybrid silver nanoparticles can be applied in the medical field.

\section{REFERENCES}

Ahmed, S., Ahmad, M., Swami, B. L., \& Ikram, S. (2016) A review on plants extract mediated synthesis of silver nanoparticles for antimicrobial applications: A green expertise, Journal of Advanced Research, 7(1), 17-28. https://doi.org/10.1016/j.jare.2015.02.007

Bhakya, S., Muthukrishnan, S., Sukumaran, M., \& Muthukumar, M. (2016). Biogenic synthesis of silver nanoparticles and their antioxidant and antibacterial activity, Applied Nanoscience, 6(5), 755-766. https://doi. org/10.1007/s13204-015-0473-z

Clogston, J. D., \& Patri, A. K. (2011). Zeta potential measurement. Methods in Molecular Biology, 697, 63-70. https://doi.org/10.1007/978-160327-198-1 6
Dawadi, S., Katuwal, S., Gupta, A., Lamichhane, U., Thapa, R., Jaisi, S. Lamichhane, G., Bhattarai, D. P., \& Parajuli, N. (2021). Current research on silver nanoparticles: synthesis, characterization, and applications. Journal of Nanomaterials, 2021, 6687290. https://doi. org/10.1155/2021/6687290

Garibo, D., Borbón-Nuñez, H. A., de León, J. N. D., Mendoza, E. G., Estrada, I., Toledano-Magaña, Y., Tiznado, H., Ovalle-Marroquin, M., Soto-Ramos, A. G., Blanco, A., Rodríguez, J. A., Romo, O. A., Chávez-Almazán, L. A., \& Susarrey-Arce, A. (2020). Green synthesis of silver nanoparticles using Lysiloma acapulcensis exhibit high-antimicrobial activity. Scientific Reports, 10, 12805. https://doi.org/10.1038/s41598-020-69606-7

Holzwarth, U., \& Gibson, N. (2011). The Scherrer equation versus the 'Debye-Scherrer equation'. Nature Nanotechnology, 6(9), 534. https:// doi.org/10.1038/nnano.2011.145

Jeena, K., Liju, V. B., \& Kuttan, R. (2013). Antioxidant, anti-inflammatory and antinociceptive activities of essential oil from ginger. Indian Journal of Physiology and Pharmacology, 57(1), 51-62.

Mangindaana, D., Linb, G-Y., Kuoc, C. J., \& Chien, H-W. (2020), Biosynthesis of silver nanoparticles as catalyst by spent coffee ground/recycled poly(ethyleneterephthalate) composites, Food and Bioproducts Processing, 121, 193-201, https://doi.org/10.1016/j.fbp.2020.02.008

Pattabiraman, S., Tamilselvan, A., Karuthapandian, S., \& Mehalingam, P. (2016). Green synthesis of silver nanoparticles, characterization, screening of phytochemicals, assessment of antioxidant and antimicrobial activity using the bark extracts of Mimusops elengi L. Energy and Environment Focus, 5(3), 200-206. https://doi. org/10.1166/eef.2016.1221

Roy, A., Bulut, O., Some, S., Mandal, A. K., \& Yilmaz, M. D. (2019), Green synthesis of silver nanoparticles: biomolecule-nanoparticle organizations targeting antimicrobial activity, Recent Scientific Advances, 9, 2773, https://doi.org/10.1039/c8ra08982e

Sanati, M., \& Andersson, A. (1993). DRIFT study of the oxidation and the ammoxidation of toluene over a $\mathrm{TiO},(\mathrm{B})$-supported vanadium catalyst. Journal of Molecular Catalysis, 81, 51-62

Sharma, A., \& Bhagatwala, J. (2019). Food, nutrients, and dietary supplements in management of disorders of gut-brain interaction, formerly functional gastrointestinal disorders. In R. R. Watson \& V. R Preedy (Eds.), Dietary Interventions in Gastrointestinal Diseases (Ch. 6, pp. 73-80), Academic Press. https://doi.org/10.1016/B978-0-12 814468-8.00006-5

Valgas, C., Souza, S. M., Smania, Elza, F. A., \& Smania Jr, A. (2007) Screening methods to determine antibacterial activity of natural products. Brazilian Journal of Microbiology, 38(2), 369-380. https:// doi.org/10.1590/S1517-83822007000200034 